\title{
Research Article (Weak) Gorenstein Global Dimension of Semiartinian Rings
}

\section{Mohammed Tamekkante and Mohamed Chhiti}

Department of Mathematics, Faculty of Science and Technology of Fez, University S.M. Ben Abdellah Fez, Morocco

Correspondence should be addressed to Mohammed Tamekkante, tamekkante@yahoo.fr

Received 18 April 2011; Accepted 20 May 2011

Academic Editors: A. Jaballah and D. Kressner

Copyright (C) 2011 M. Tamekkante and M. Chhiti. This is an open access article distributed under the Creative Commons Attribution License, which permits unrestricted use, distribution, and reproduction in any medium, provided the original work is properly cited.

We prove that if $R$ is a semiartinian commutative ring, the Gorenstein global dimension of $R$ equals the supremum of the Gorenstein projective and injective dimensions of simple $R$-modules, and the weak Gorenstein global dimension of $R$ equals the supremum of the Gorenstein flat dimensions of simple $R$-modules.

\section{Introduction}

Throughout the paper, all rings are commutative with identity. Let $R$ be a ring, and let $M$ be an $R$-module. As usual, we use $\operatorname{pd}_{R}(M), \mathrm{id}_{R}(M)$, and $\mathrm{fd}_{R}(M)$ to denote, respectively, the classical projective dimension, injective dimension, and flat dimension of $M$.

For a two-sided Noetherian ring $R$, Auslander and Bridger [1] introduced the $G$-dimension, $\operatorname{Gdim}_{R}(M)$, for every finitely generated $R$-module $M$. They showed that $\operatorname{Gdim}_{R}(M) \leq \operatorname{pd}_{R}(M)$ for all finitely generated $R$-modules $M$, and equality holds if $\operatorname{pd}_{R}(M)$ is finite.

Several decades later, Enochs and Jenda [2,3] introduced the notion of Gorenstein projective dimension ( $G$-projective dimension for short), as an extension of $G$ dimension to modules that are not necessarily finitely generated, and the Gorenstein injective dimension (G-injective dimension for short) as a dual notion of Gorenstein projective dimension. Then, to complete the analogy with the classical homological dimension, Enochs et al. [4] introduced the Gorenstein flat dimension. Some references are [2-8].

Recall that an $R$-module $M$ is called Gorenstein projective, if there exists an exact sequence of projective $R$-modules

$$
\mathbf{P}: \cdots \longrightarrow P_{1} \longrightarrow P_{0} \longrightarrow P^{0} \longrightarrow P^{1} \longrightarrow \cdots
$$


such that $M \cong \operatorname{Im}\left(P_{0} \longrightarrow P^{0}\right)$ and such that the functor $\operatorname{Hom}_{R}(-, Q)$ leaves $\mathbf{P}$ exact whenever $Q$ is a projective $R$-module. The complex $\mathbf{P}$ is called a complete projective resolution.

The Gorenstein injective $R$-modules are defined dually.

An $R$-module $M$ is called Gorenstein flat, if there exists an exact sequence of flat $R$ modules:

$$
\mathbf{F}: \cdots \longrightarrow F_{1} \longrightarrow F_{0} \longrightarrow F^{0} \longrightarrow F^{1} \longrightarrow \cdots
$$

such that $M \cong \operatorname{Im}\left(F_{0} \longrightarrow F^{0}\right)$ and such that the functor $I \otimes_{R^{-}}$leaves $\mathbf{F}$ exact whenever $I$ is a right injective $R$-module. The complex $\mathrm{F}$ is called a complete flat resolution.

The Gorenstein projective, injective, and flat dimensions are defined in terms of resolutions and denoted by $\operatorname{Gpd}(-), \operatorname{Gid}(-)$, and $\operatorname{Gfd}(-)$, respectively (see $[6,8,9])$.

In [5], for any associative ring $R$, the authors proved the equality

$$
\sup \left\{\operatorname{Gpd}_{R}(M) \mid M \text { is a (left) } R \text {-module }\right\}=\sup \left\{\operatorname{Gid}_{R}(M) \mid M \text { is a (left) } R \text {-module }\right\} \text {. }
$$

They called the common value of the above quantities the left Gorenstein global dimension of $R$ and denoted it by $l$.Ggldim $(R)$. Similarly, they set

$$
\text { l.wGgldim }(R)=\sup \left\{\operatorname{Gfd}_{R}(M) \mid M \text { is a (left) } R \text {-module }\right\} \text {, }
$$

which they called the left Gorenstein weak dimension of $R$. Since all rings in this paper are commutative, we drop the letter $l$.

Recall that an $R$-module $M$ is called semiartinian, if every nonzero quotient module of $M$ has nonzero socle. A ring $R$ is said to be semiartinian if it is semiartinian as an $R$-module; see [10].

In [11], the authors characterized the (resp., weak) Gorenstein global dimension for an arbitrary associative ring. The purpose of this paper is to apply these characterizations to a commutative semiartinian rings. Hence, we prove that if $R$ is a semiartinian commutative ring, the Gorenstein global dimension of $R$ equals the supremum of the Gorenstein projective and injective dimension of simple $R$-modules (Theorem 2.1), and the weak Gorenstein global dimension of $R$ equals the supremum of the Gorenstein flat dimensions of simple $R$-modules (Theorem 2.7).

\section{Main Results}

The first main result of this paper computes the Gorenstein global dimension of semiartinian rings via the Gorenstein projective and injective dimensions of simple modules.

Theorem 2.1. Let $R$ be a semiartinian ring and $n$ a positive integer. The following conditions are equivalent:

(1) $\operatorname{Gg} \operatorname{ldim}(R) \leq n$,

(2) $\sup _{C}\left\{\operatorname{Gpd}_{R}(C), \operatorname{Gid}_{R}(C)\right\} \leq n$ where $C$ ranges ranges over all simple $R$-modules,

(3) $\operatorname{Ext}_{R}^{n+1}(C, P)=\operatorname{Tor}_{R}^{n+1}(I, C)=0$ for all simple $R$-modules $C$, all projective $R$-modules $P$, and all injective $R$-modules $I$. 
Consequently,

$$
\operatorname{Ggldim}(R)=\sup _{C}\left\{\operatorname{Gpd}_{R}(C), \operatorname{Gid}_{R}(C)\right\}
$$

where $C$ ranges ranges over all simple $R$-modules.

To prove this theorem, we need the following lemma.

Lemma 2.2 (Theorem 2.1, [11]). Let $R$ be a ring and $n$ a positive integer. Then, $\operatorname{Ggldim}(R) \leq n$ if, and only if, $R$ satisfies the following two conditions:

$(\mathrm{C} 1)$ : $\operatorname{id}(P) \leq n$ for every projective $R$-module $P$,

(C2): $\operatorname{pd}(I) \leq n$ for every injective $R$-module $I$.

Proof of Theorem 2.1. (1) $\Rightarrow$ (2) Clear by the definition of $\operatorname{Ggldim}(R)$.

$(2) \Rightarrow(3)$ By [8, Theorem 2.20], $\operatorname{Ext}_{R}^{n+1}(C, P)=0$ for all $i>n$ and all simple $R$-module $C$ and all projective module $P$ since $\operatorname{Gpd}_{R}(C) \leq n$. Let $I$ be an injective $R$-module. By [8, Theorem 2.22], $\operatorname{Ext}_{R}^{n+1}(I, C)=0$ for all $i>n$ and all simple $R$-module $C$ since $\operatorname{Gid}_{R}(C) \leq n$. Then, by [12, Lemma 3.1(1)], $\operatorname{Tor}_{R}^{n+1}(I, C)=0$ for every simple $R$-module $C$.

$(3) \Rightarrow(1)$ Let $P$ be a projective $R$-module. By [12, Lemma 4.2(2)], $\operatorname{id}_{R}(P) \leq n$ since $\operatorname{Ext}_{R}^{n+1}(C, P)=0$ for all simple $R$-modules $C$. Hence, the condition (C1) of Lemma 2.2 is clear. Let now $I$ be an arbitrary injective $R$-module. By [12, Lemma 4.2(1)], $\mathrm{fd}_{R}(I) \leq n$ since $\operatorname{Tor}_{R}^{n+1}(I, C)=0$ for all simple $R$-module $C$. On the other hand, from [13, Theorem 7.2.5(2)

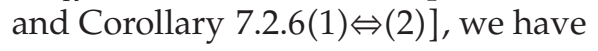

$$
\begin{aligned}
(a) & :=\sup \left\{\operatorname{pd}_{R}(F) \mid F \text { is a flat } R \text {-module }\right\} \\
& \leq(b):=\sup \left\{\operatorname{pd}_{R}(M) \mid M \text { is an } R \text {-module with } \operatorname{pd}_{R}(M)<\infty\right\} \\
& \leq(c):=\sup \left\{\operatorname{id}_{R}(P) \mid P \text { is a projective } R \text {-module }\right\} .
\end{aligned}
$$

Moreover, we have just proved that $(c) \leq n$, and so $(a) \leq n$. Accordingly, since $\mathrm{fd}_{R}(I) \leq n$, we have $\operatorname{pd}_{R}(I)<\infty$. Hence, since $(b) \leq n$, we get $\operatorname{pd}_{R}(I) \leq n$. Consequently, the condition (C2) of Lemma 2.2 is clear. As consequence, $\operatorname{Ggldim}(R) \leq n$, as desired.

Remark 2.3. From the proof of Theorem 2.1, we can easily see that

$$
\sup \left\{\operatorname{id}_{R}(P) \mid P \text { is a projective } R \text {-module }\right\} \leq \sup \left\{\operatorname{Gpd}_{R}(C) \mid C \text { is a simple } R \text {-module }\right\},
$$

provided $R$ is a semiartinian ring.

Corollary 2.4. Let $R$ be a semiartinian ring with finite Gorenstein global dimension. Then,

$$
\operatorname{Ggldim}(R)=\sup _{C}\left\{\operatorname{Gpd}_{R}(C)\right\} \text {, }
$$

where $C$ ranges over all simple $R$-modules. 
Proof. It is sufficient to prove the inequality $\operatorname{Ggldim}(R) \leq \sup _{C}\left\{\operatorname{Gpd}_{R}(C)\right\}$, where $C$ ranges over all simple $R$-modules. We may suppose $n=\sup _{C}\left\{\operatorname{Gpd}_{R}(C)\right\}$ finite. By Remark 2.3, $\sup \left\{\operatorname{id}_{R}(P) \mid P\right.$ is a projective $R$-module $\} \leq n$. Using [8, Theorem 2.20], $\operatorname{Gpd}_{R}(M) \leq n$ for any $R$-module $M$. Consequently, $\operatorname{Ggldim}(R) \leq n$, as desired.

Recall that ring $R$ is called quasi-Frobenius if it is self injective and artinian.

Corollary 2.5. The following conditions are equivalent:

(1) $R$ is quasi-Frobenius,

(2) $R$ is a semiartinian ring and every simple $R$-module is Gorenstein projective.

Proof. (1) $\Rightarrow$ (2) Follows immediately from [5, Proposition 2.10] and Corollary 2.4.

$(2) \Rightarrow(1)$ From Remark 2.3, every projective module is injective. Thus, by [14, Theorem 7.55], $R$ is quasi-Frobenius. corollary.

Since every perfect ring is semiartinian by [10, Proposition 5.1], we have the following

Corollary 2.6. If $R$ is a perfect ring with Jacobson radical $J$, then

$$
\operatorname{Ggldim}(R)=\sup \left\{\operatorname{Gpd}_{R}\left(\frac{R}{J}\right), \operatorname{Gid}_{R}\left(\frac{R}{J}\right)\right\}
$$

Moreover, if $R$ is not quasi-Frobenius ring then, $\operatorname{Ggldim}(R)=1+\sup \left\{\operatorname{Gpd}_{R}(J), \operatorname{Gid}_{R}(J)\right\}$.

Proof. Using [8, Proposition 2.19] and its dual version, this result is immediate since every simple $R$-module is a direct summand of the $R$-module $R / J$ by [15, Theorem 9.3.4].

The second main result of this paper computes the weak Gorenstein global dimension of semiartinian rings via the Gorenstein flat dimensions of simple modules.

Theorem 2.7. Let $R$ be a semiartinian ring and let $n$ be a positive integer. The following conditions are equivalent:

(1) $\mathrm{wGgldim}(R) \leq n$,

(2) $\operatorname{Gfd}_{R}(C) \leq n$ for all simple $R$-modules $C$,

(3) $\operatorname{Tor}_{R}^{n+1}(I, C)=0$ for all simple $R$-modules $C$ and all injective $R$-modules $I$.

Consequently, $\mathrm{wGgldim}(R)=\sup _{C}\left\{\operatorname{Gfd}_{R}(C)\right\}$, where $C$ ranges ranges over all simple $R$-modules.

We need the following lemmas.

Lemma 2.8. Let $M$ be an $R$-module. If $\operatorname{Gfd}_{R}(M) \leq n$ then $\operatorname{Tor}_{R}^{i}(I, M)=0$ for all $i>n$ and every injective $R$-modules $I$.

Proof. Using the definition of Gorenstein flat module, the case $n=0$ is clear. For $n>0$, we consider an $n$-step flat resolution of $M$, and we use the start case.

Lemma 2.9 (Theorem 2.4, [11]). Let $R$ be a ring and $n$ a positive integer. The following conditions are equivalent: 
(1) $w \operatorname{Ggldim}(R) \leq n$,

(2) $\mathrm{fd}_{R}(I) \leq n$ for every injective module $I$.

Proof of Theorem 2.7. (1) $\Rightarrow(2)$ Follows immediately from the definition of weak Gorenstein global dimension.

$(2) \Rightarrow(3)$ Follows from Lemma 2.8 .

$(3) \Rightarrow(1)$ By $[12$, Lemma $4.2(1)], \mathrm{fd}_{R}(I) \leq n$ for every injective $R$-module $I$ since $\operatorname{Tor}_{R}^{n+1}(I, C)=0$ for all simple $R$-modules $C$. Hence, this implication follows from Lemma 2.9.

Recall that a ring is called IF-ring if every injective module is flat; see [16].

Corollary 2.10. Let $R$ be a semiartinian ring. The following are equivalent:

(1) $R$ is an IF-ring.

(2) Every simple R-module is Gorenstein flat.

(3) $R$ is coherent and $E(C)$ is flat for every simple $R$-module $C$ where $E(C)$ is the injective envelope of $C$.

Proof. Using Lemma 2.8, a ring $R$ is $I F$-ring if and only if $\operatorname{wGgldim}(R)=0$. Hence, the equivalence $(1) \Leftrightarrow(2)$ is an immediate consequence of Theorem 2.7.

(3) $\Rightarrow(1)$ Follows from [16, Theorem 3.8].

$(1) \Rightarrow(3)$ From Lemma 2.9, if $R$ is an $I F$-ring, then every $R$-module is Gorenstein flat. Then, by [17, Theorem 6], $R$ is coherent and self $F P$-injective (i.e., $\operatorname{Ext}_{R}^{1}(M, R)=0$ for every finitely presented $R$-module $M$ ). Thus, by [16, Theorem 3.8], $E(C)$ is flat for every simple $R$-module $C$, where $E(C)$ is the injective envelope of $C$, and certainly $R$ is coherent.

Remark 2.11. Note that the equivalence of (1) and (3) in the above corollary does not need that $R$ be semiartinian, see [16, Theorem 3.8] and [18, Proposition 4.2].

Lemma 2.12. Let $0 \longrightarrow N \longrightarrow N^{\prime} \longrightarrow N^{\prime \prime} \longrightarrow 0$ be an exact sequence of modules over a coherent ring $R$. Then, $\operatorname{Gpd}_{R}\left(N^{\prime \prime}\right) \leq \max \left\{\operatorname{Gpd}_{R}\left(N^{\prime}\right), \operatorname{Gpd}_{R}(N)+1\right\}$ with equality if $\operatorname{Gpd}_{R}\left(N^{\prime}\right) \neq \operatorname{Gpd}_{R}(N)$.

Proof. Using [8, Theorem 3.15] and [8, Theorem 3.14], the proof is similar to that of [19, Corollary 2, page 135].

Corollary 2.13. If $R$ is a perfect coherent ring with Jacobson radical $J$, then $\operatorname{wGgldim}(R)=$ $\operatorname{Gfd}_{R}(R / J)$. Moreover, if $R$ is not IF-ring then, $\operatorname{wGgldim}(R)=1+\sup \left\{\operatorname{Gfd}_{R}(J)\right\}$.

Proof. Using [8, Proposition 3.13], the first statement of this result is an immediate consequence of Theorem 2.7 since every simple $R$-module is a direct summand of the $R$ module $R / J$ by [15, Theorem 9.3.4].

Suppose that $R$ is not $I F$-ring. Then, by Lemma 2.9, $\operatorname{wGgldim}(R)=\operatorname{Gfd}_{R}(R / N)>0$. Therefore, by Lemma 2.12, we deduce from the exact sequence

$$
0 \longrightarrow N \longrightarrow R \longrightarrow \frac{R}{N} \longrightarrow 0
$$

that $\mathrm{wGgldim}(R)=\operatorname{Gpd}_{R}(R / N)=1+\operatorname{Gpd}_{R}(N)$. 


\section{Acknowledgment}

The authors would like to express their sincere thanks to the referees for their helpful suggestions and comments.

\section{References}

[1] M. Auslander and M. Bridger, Stable Module Theory, Memoirs of the American Mathematical Society, No. 94, American Mathematical Society, Providence, RI, USA, 1969.

[2] E. Enochs and O. Jenda, "On Gorenstein injective modules," Communications in Algebra, vol. 21, no. 10, pp. 3489-3501, 1993.

[3] E. Enochs and O. Jenda, "Gorenstein injective and projective modules," Mathematische Zeitschrift, vol. 220, no. 4, pp. 611-633, 1995.

[4] E. Enochs, O. Jenda, and B. Torrecillas, "Gorenstein flat modules," Nanjing Daxue Xuebao Shuxue Bannian Kan, vol. 10, no. 1, pp. 1-9, 1993.

[5] D. Bennis and N. Mahdou, "Global Gorenstein dimensions," Proceedings of the American Mathematical Society, vol. 138, no. 2, pp. 461-465, 2010.

[6] L. W. Christensen, Gorenstein Dimensions, vol. 1747 of Lecture Notes in Mathematics, Springer, Berlin, Germany, 2000.

[7] L. W. Christensen, A. Frankild, and H. Holm, "On Gorenstein projective, injective and flat dimensions-a functorial description with applications," Journal of Algebra, vol. 302, no. 1, pp. 231$279,2006$.

[8] H. Holm, "Gorenstein homological dimensions," Journal of Pure and Applied Algebra, vol. 189, no. 1-3, pp. 167-193, 2004.

[9] E. Enochs and O. Jenda, Relative Homological Algebra, de Gruyter Expositions in Mathematics, Walter de Gruyter \& Company, Berlin, Germany, 2000.

[10] B. Stenstrom, Rings of Quotients, Springer, New York, NY, USA, 1975.

[11] N. Mahdou and M. Tamekkante, "Note on (weak) Gorenstein global dimension".

[12] N. Ding and J. Chen, "The homological dimensions of simple modules," Bulletin of the Australian Mathematical Society, vol. 48, no. 2, pp. 265-274, 1993.

[13] L. Mao and N. Ding, "The cotorsion dimension of modules and rings," in Abelian Groups, Rings, Modules, and Homological Algebra, vol. 249 of Lecture Notes in Pure Applied Mathematics, pp. 217-233, Chapman \& Hall/CRC, Boca Raton, Fla, USA, 2006.

[14] W. K. Nicholson and M. F. Yousif, Quasi-Frobenius Rings, vol. 158 of Cambridge Tracts in Mathematics, Cambridge University Press, Cambridge, NY, USA, 2003.

[15] F. Kasch, Modules and Rings, London Mathematical Society Monographs, Academic Press, London, UK, 1982.

[16] N. Ding and J. Chen, "The flat dimensions of injective modules," Manuscripta Mathematica, vol. 78, no. 2, pp. 165-177, 1993.

[17] N. Ding and J. Chen, "Coherent rings with finite self ${ }^{F P}$-injective dimension," Communications in Algebra, vol. 24, no. 9, pp. 2963-2980, 1996.

[18] B. Stenstrom, "Coherent rings and ${ }^{F P}$-injective modules," Journal of the London Mathematical Society, vol. 2, pp. 323-329, 1970.

[19] N. Bourbaki, Éléments de Mathématique, Algébre Homologique, Chapitre 10, Masson, Paris, France, 1980. 


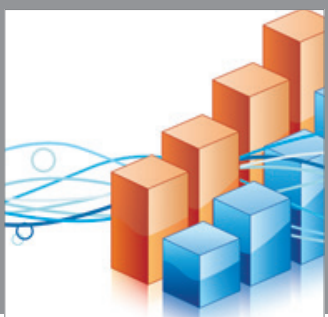

Advances in

Operations Research

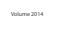

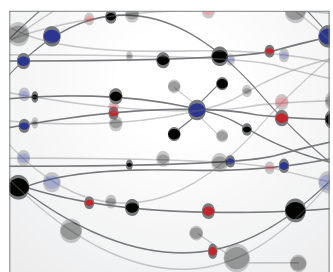

\section{The Scientific} World Journal
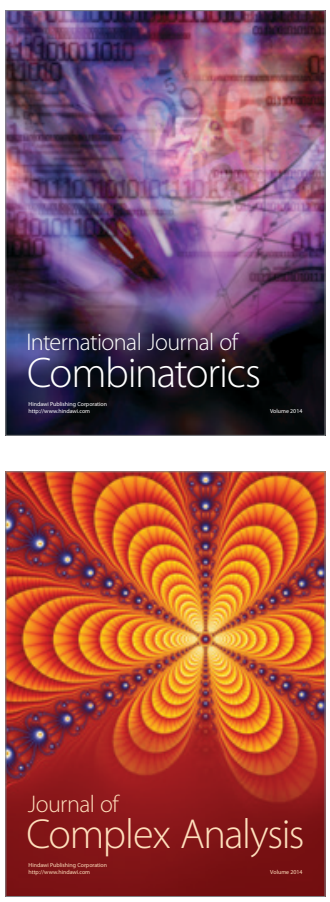

International Journal of

Mathematics and

Mathematical

Sciences
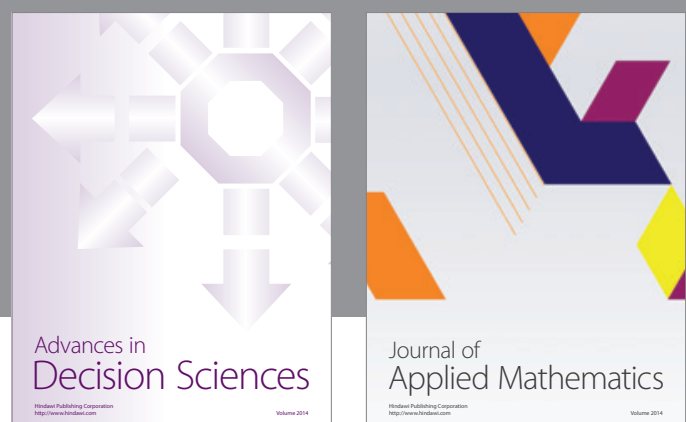

Journal of

Applied Mathematics
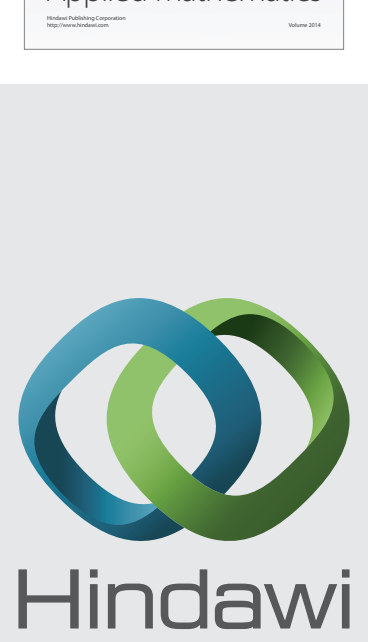

Submit your manuscripts at http://www.hindawi.com
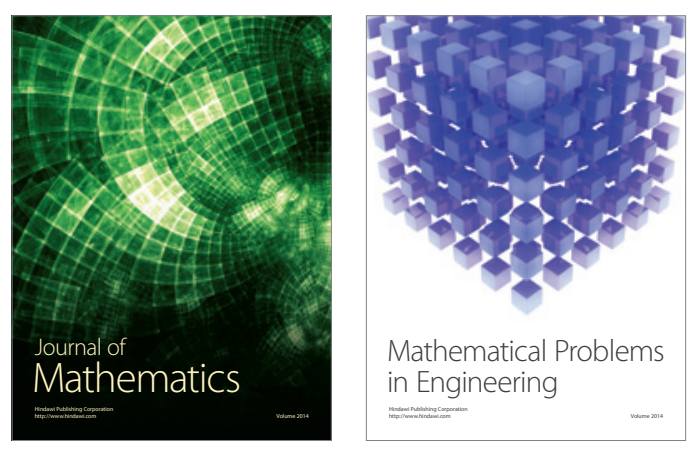

Mathematical Problems in Engineering
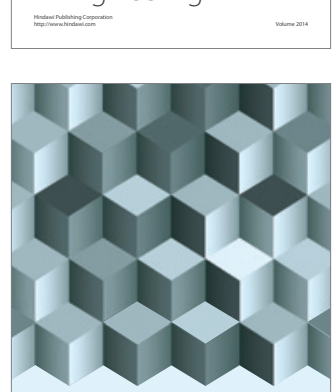

Journal of

Function Spaces
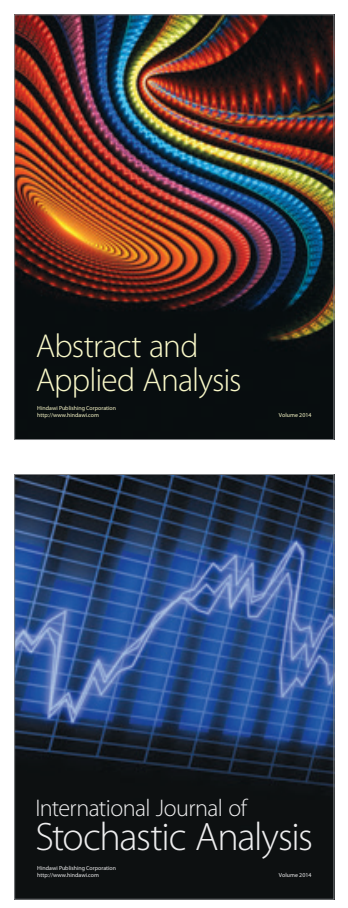

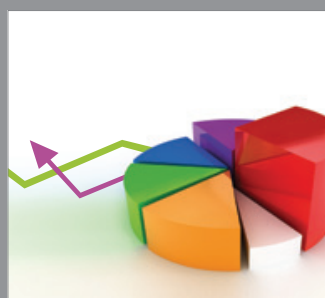

ournal of

Probability and Statistics

Promensencen
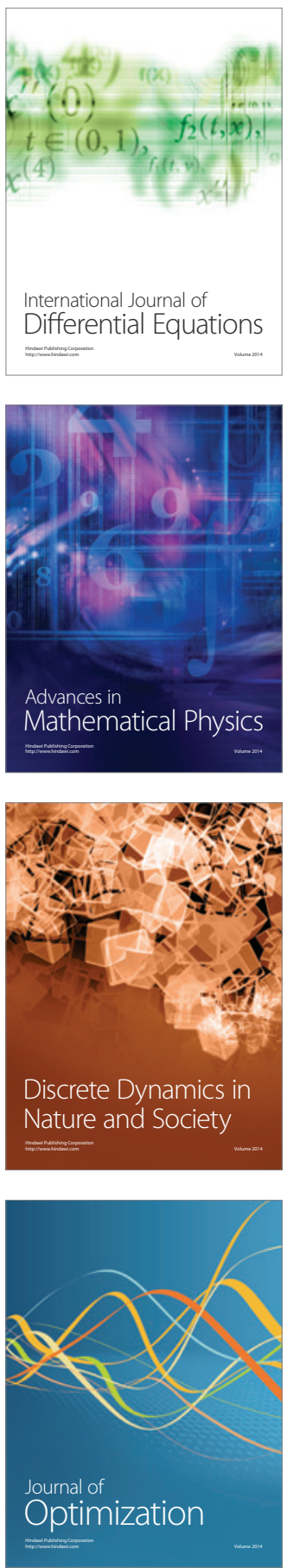